\title{
Quantum Imaging beyond the Diffraction Limit by Optical Centroid Measurements
}

\author{
Mankei Tsang* \\ Research Laboratory of Electronics, Massachusetts Institute of Technology, Cambridge, Massachusetts 02139, USA
}

(Received 9 March 2009; published 22 June 2009)

\begin{abstract}
I propose a quantum imaging method that can beat the Rayleigh-Abbe diffraction limit and achieve de Broglie resolution without requiring a multiphoton absorber or coincidence detection. Using the same nonclassical states of light as those for quantum lithography, the proposed method requires only optical intensity measurements, followed by image postprocessing, to produce the same complex quantum interference patterns as those in quantum lithography. The method is expected to be experimentally realizable using current technology.
\end{abstract}

DOI: 10.1103/PhysRevLett.102.253601

PACS numbers: 42.50.St, 42.50.Dv

It has been suggested that an ensemble of photons, under appropriate measurements, can be regarded as a single quantum object with a much smaller effective wavelength, called the photonic de Broglie wavelength [1]. The de Broglie wavelength of $N$ photons, each with classical wavelength $\lambda$, can be as small as $\lambda / N$. It is especially desirable for imaging applications to take advantage of the small de Broglie length scale, since the resolution of classical optical imaging is limited by the size of $\lambda$, according to the Rayleigh-Abbe diffraction limit [2]. A seminal paper by Boto et al. suggests that the $N$-photon absorption patterns of certain nonclassical $N$-photon states exhibit de Broglie resolution [3]. Subsequent work by various researchers on quantum imaging has sought to improve upon the original "quantum lithography" technique [4-8], yet all of these proposals still require a multiphoton absorber as the detector [4-6,8] or coincidence detection [7]. The low efficiency of $N$-photon absorption and coincidence detection, especially for large $N$, is a significant obstacle to the use of quantum technology in real-world imaging applications.

In this Letter, I propose a quantum imaging method that achieves de Broglie resolution without requiring a multiphoton absorber or coincidence detection. I shall show how the same images as those in quantum lithography can be reconstructed simply by intensity measurements, followed by image postprocessing. Given the availability of highefficiency single-photon detectors, the proposed method has the potential to beat the diffraction limit by a large amount using current technology.

A different way of achieving de Broglie resolution is via optical-beam displacement measurements, using a split detector or homodyne detection, as investigated by Fabre and co-workers [9]. The standard quantum limit to the displacement uncertainty is on the order of $\Delta x / \sqrt{N}$, where $\Delta x$ is the classical beam width, while the "Heisenberg" limit is on the order of $\Delta x / N$ [9]. The quantum imaging theory I propose here can be considered as a generalization of Fabre et al.'s results, and therefore presents a unified view of different quantum strategies that take advantage of the photonic de Broglie wavelength. I show that the displacement measurements can not only be considered as a Gaussian single-parameter estimation technique, but repeated measurements of the parameter can also yield complex quantum interference patterns identical to those in quantum lithography. The proposed method thus provides an accessible way of detecting nonclassical and nonGaussian signatures of multimode quantum optical states, and is expected to become an important tool in the study of quantum optics and quantum physics in general.

I shall first briefly review the configuration-space theory of quantum lithography [10]. For simplicity, I consider only free-space photons observed at the image plane in one transverse dimension, $x$, in the paraxial regime. Generalization to two transverse dimensions and the nonparaxial regime is possible $[10,11]$, but does not add much insight, and the results should remain qualitatively the same. Let $\hat{a}(k)$ and $\hat{a}^{\dagger}(k)$ be the photon annihilation and creation operators, respectively, in the transversemomentum space, with the bosonic commutation relation $\left[\hat{a}(k), \hat{a}^{\dagger}\left(k^{\prime}\right)\right]=\delta\left(k-k^{\prime}\right)$. First, consider a pure $N$-photon quantum state $|N\rangle$. The multiphoton momentum eigenket is $\left|k_{1}, \ldots, k_{N}\right\rangle \equiv(N !)^{-1 / 2} \hat{a}^{\dagger}\left(k_{1}\right) \ldots \hat{a}^{\dagger}\left(k_{N}\right)|0\rangle$. The momentum wave function representation of $|N\rangle$ is then

$$
\phi\left(k_{1}, \ldots, k_{N}\right) \equiv\left\langle k_{1}, \ldots, k_{N} \mid N\right\rangle .
$$

The Rayleigh-Abbe diffraction limit [2] restricts the transverse momenta of photons to a finite bandwidth:

$$
\phi\left(k_{1}, \ldots, k_{N}\right)=0 \text { for any }\left|k_{n}\right|>\frac{2 \pi \sin \theta}{\lambda},
$$

where $\sin \theta$ is the numerical aperture of the optical system and $\sin \theta \ll 1$ defines the paraxial regime.

The spatial annihilation operator is defined as $\hat{A}(x) \equiv$ $(2 \pi)^{-1 / 2} \int d k \hat{a}(k) \exp (i k x)$ and can be used to construct $N$-photon states with definite positions $\left|x_{1}, \ldots, x_{N}\right\rangle \equiv$ $(N !)^{-1 / 2} \hat{A}^{\dagger}\left(x_{1}\right) \ldots \hat{A}^{\dagger}\left(x_{N}\right)|0\rangle$. A multiphoton-position positive operator-valued measure (POVM) can be defined as 


$$
\hat{\Pi}\left(x_{1}, \ldots, x_{N}\right)=\left|x_{1}, \ldots, x_{N}\right\rangle\left\langle x_{1}, \ldots, x_{N}\right|,
$$

with normalization $\int d x_{1} \ldots d x_{N} \hat{\Pi}\left(x_{1}, \ldots, x_{N}\right)=\hat{1}$. The position wave function is then

$$
\psi\left(x_{1}, \ldots, x_{N}\right) \equiv\left\langle x_{1}, \ldots, x_{N} \mid N\right\rangle,
$$

which is the $N$-dimensional Fourier transform of $\phi\left(k_{1}, \ldots, k_{N}\right)$. Changing the position variables to the centroid and relative-position coordinates, defined as

$$
X \equiv \frac{1}{N} \sum_{n=1}^{N} x_{n}, \quad \xi_{n} \equiv x_{n}-X,
$$

respectively, a new POVM and a new wave function are obtained:

$$
\begin{gathered}
\hat{\Upsilon}\left(X, \xi_{1}, \ldots, \xi_{N-1}\right) \equiv N \hat{\Pi}\left(X+\xi_{1}, \ldots, X+\xi_{N}\right), \\
f\left(X, \xi_{1}, \ldots, \xi_{N-1}\right) \equiv \sqrt{N} \psi\left(X+\xi_{1}, \ldots, X+\xi_{N}\right),
\end{gathered}
$$

where $\xi_{N}=-\sum_{n=1}^{N-1} \xi_{n}$. It is not difficult to show that the $N$-photon-absorption probability distribution of the $N$-photon state $\hat{\rho}_{N} \equiv|N\rangle\langle N|$ is given by

$$
\begin{aligned}
\left\langle: \hat{I}^{N}(x):\right\rangle & =\left\langle\left[\hat{A}^{\dagger}(x)\right]^{N}[\hat{A}(x)]^{N}\right\rangle \propto \operatorname{Tr}\left[\hat{\Upsilon}(x, 0, \ldots, 0) \hat{\rho}_{N}\right] \\
& =|f(x, 0, \ldots, 0)|^{2},
\end{aligned}
$$

which is proportional to the conditional centroid probability distribution $p_{c}(x) \equiv \operatorname{Tr}\left[\hat{\Upsilon}(x, 0, \ldots, 0) \hat{\rho}_{N}\right] /$ $\int d x \operatorname{Tr}\left[\hat{Y}(x, 0, \ldots, 0) \hat{\rho}_{N}\right]$, given that all relative positions are zero. The variable conjugate to the centroid position $X$ is the total momentum $K \equiv \sum_{n=1}^{N} k_{n}$. The bandwidth limit on $K$ becomes $|K| \leq 2 \pi N \sin \theta / \lambda$, so the minimum feature size of the centroid distribution is on the order of $\lambda /(2 N \sin \theta)$. See Fig. 1 for an illustration of the configuration-space theory.

Multiphoton absorption is not the only way of accessing the centroid degree of freedom. Consider the intensity centroid operator,

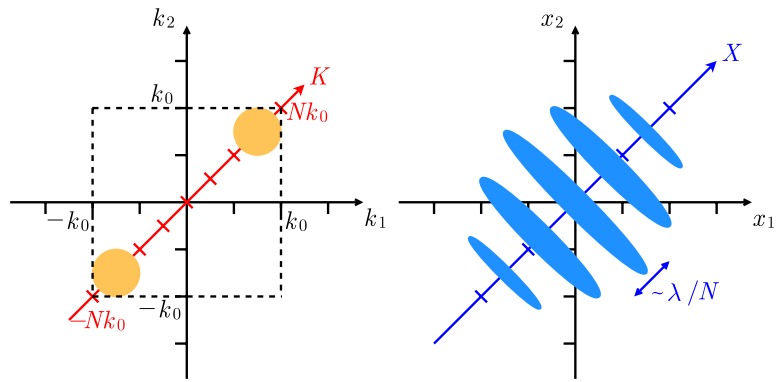

FIG. 1 (color online). Configuration-space picture of quantum imaging for $N=2$. The Rayleigh-Abbe diffraction limit restricts the momentum wave function inside an $N$-dimensional box $\left|k_{n}\right| \leq k_{0} \equiv 2 \pi \sin \theta / \lambda$. The magnitude of the total momentum $K=\sum_{n=1}^{N} k_{n}$ is then limited by $|K| \leq N k_{0}$. The minimum feature size of the wave function with respect to the centroid coordinate $X$ is then $\sim 1 /\left(N k_{0}\right) \sim \lambda / N$.

$$
\hat{X} \equiv \frac{1}{N} \int d x x \hat{A}^{\dagger}(x) \hat{A}(x)
$$

which can be measured by spatially resolving intensity measurements, using, for example, a photon-counting detector array, followed by a calculation of the centroid of the intensity pattern, as shown schematically in Fig. 2. The effect of finite detector pixel size is to discretize the image plane or equivalently limit the spatial bandwidth of the photons by virtue of the sampling theorem, so the size of each pixel should be much smaller than $\lambda /(2 \sin \theta)$. If the pixel size is small enough, such that the probability of more than one photon falling on each is much smaller than one, single-photon detectors can be used instead. The intensity operator can be rewritten as

$$
\begin{aligned}
\hat{X} & =\hat{X} \hat{1}=\hat{X} \int d x_{1} \ldots d x_{N}\left|x_{1}, \ldots, x_{N}\right\rangle\left\langle x_{1}, \ldots, x_{N}\right| \\
& =\int d x_{1} \ldots d x_{N}\left(\frac{1}{N} \sum_{n=1}^{N} x_{n}\right) \hat{\Pi}\left(x_{1}, \ldots, x_{N}\right) \\
& =\int d X d \xi_{1} \ldots d \xi_{N-1} X \hat{Y}\left(X, \xi_{1}, \ldots, \xi_{N-1}\right) .
\end{aligned}
$$

Thus, measurements of the intensity centroid realizes the marginal POVM

$$
\hat{\Upsilon}(X) \equiv \int d \xi_{1} \ldots d \xi_{N-1} \hat{\Upsilon}\left(X, \xi_{1}, \ldots, \xi_{N-1}\right),
$$

and the probability distribution of the intensity centroid
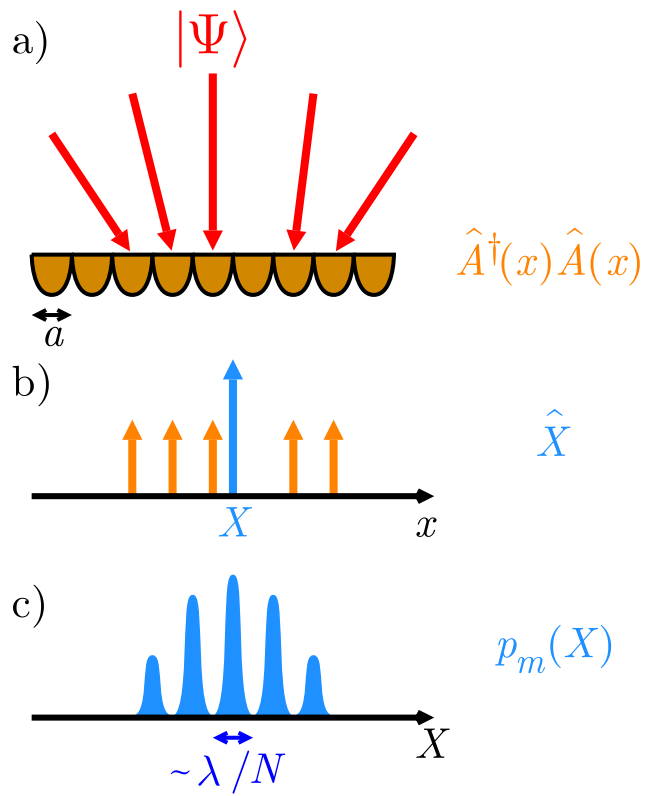

FIG. 2 (color online). (a) To measure the intensity centroid distribution, first make an ideal spatially resolving intensity measurement $\left[\hat{A}^{\dagger}(x) \hat{A}(x)\right]$. This can be done by, for example, an array of photon-counting detectors, each with size $a \ll$ $\lambda /(2 \sin \theta)$. (b) The centroid $X$ is calculated from the measured intensity pattern. (c) The intensity centroid distribution $p_{m}(X)$ can then be obtained after repeated measurements of $X$. 
measurements is

$$
\operatorname{Tr}\left[\hat{Y}(X) \hat{\rho}_{N}\right]=\int d \xi_{1} \ldots d \xi_{N-1}\left|f\left(X, \xi_{1}, \ldots, \xi_{N-1}\right)\right|^{2}
$$

which is the marginal centroid probability distribution, hereafter denoted as $p_{m}(X)$. The minimum feature size of the marginal centroid probability distribution is similarly limited by the bandwidth of the total momentum $|K| \leq$ $2 \pi N \sin \theta / \lambda$, and can therefore also reach the de Broglie length scale $\sim \lambda / N$. Unlike quantum lithography, which requires an $N$-photon absorber for an $N$-fold resolution enhancement, intensity centroid measurements require only photon counting and postprocessing to achieve the $N$-fold resolution enhancement for an arbitrary number of photons. Moreover, as the probability distribution of intensity centroid measurements is marginal and not conditioned upon specific values of the relative positions, the efficiency of intensity centroid measurements is fundamentally higher than that of multiphoton absorption or any other coincidence detection method. This is the central result of this Letter.

In general, the conditional centroid distribution, $p_{c}(x)$, obtained by multiphoton absorption, and the marginal centroid distribution, $p_{m}(X)$, obtained by intensity centroid measurements, are not the same, except for certain special quantum states. One such class of states are the momentum-correlated or position-anti-correlated states, of which the "NOON" state $\left(|\Psi\rangle \propto|N\rangle_{k}|0\rangle_{-k}+\right.$ $|0\rangle_{k}|N\rangle_{-k}$ ) is a famous example [3,10]. These states have small uncertainties in the relative momenta $\left(\kappa_{n} \equiv k_{n}-K\right)$ and large uncertainties in the relative positions $\xi_{n}$, such that the wave function can be approximated as

$$
f\left(X, \xi_{1}, \ldots, \xi_{N-1}\right) \approx g(X),
$$

and $p_{c}(X) \approx p_{m}(X) \approx|g(X)|^{2} \cdot p_{c}(X)$ and $p_{m}(X)$ become the magnitude squared of a complex wave function, and can therefore exhibit the same interference patterns as those in classical coherent imaging. The momentumcorrelated states are also the ones that achieve de Broglie resolution, since the bandwidth of the relative momenta $\kappa_{n}$ is negligible, allowing the bandwidth of the total momentum $K$ to reach its maximum value $\sim 2 \pi N \sin \theta / \lambda$ [10]. Such states can be created, for example, by spontaneous parametric down conversion, and have been experimentally demonstrated by D'Angelo et al. [5]. The timedomain version of momentum-correlated states has also been studied theoretically and experimentally by Giovannetti and co-workers [12].

Another class of quantum states with identical $p_{c}(X)$ and $p_{m}(X)$ are the ones with separable wave functions, as follows:

$$
f\left(X, \xi_{1}, \ldots, \xi_{N-1}\right)=g(X) h\left(\xi_{1}, \ldots, \xi_{N-1}\right),
$$

of which the quantum Gaussian beams [10] and quantum solitons [13] are notable examples. Classical Gaussian beams are a special instance of quantum Gaussian beams, and both multiphoton absorption and intensity centroid measurements of a classical Gaussian beam produce a Gaussian spot with a width on the order of $\lambda /(\sqrt{N} \sin \theta)$, as one would expect from a classical theory. At the "Heisenberg" limit, the width of the centroid distribution of quantum Gaussian beams is on the order of $\lambda /(N \sin \theta)$. One can use the quantum Gaussian beam centroid as a "laser pointer" to transmit and reconstruct an image in a point-by-point fashion $[6,8,9]$.

Next, I shall generalize the preceding results to quantum states with indefinite photon numbers and show that the effect of indefinite photon numbers does not significantly affect pattern formation by intensity centroid measurements. Define a pure state as $|\Psi\rangle=\sum_{N=0}^{\infty} C_{N}|N\rangle$, and denote the wave function of each Fock-state component by a subscript $N$. The $M$-photon absorption distribution is

$$
\begin{aligned}
& \left\langle: \hat{I}^{M}(x):\right\rangle \propto \sum_{N=M}^{\infty}\left(\begin{array}{c}
N \\
M
\end{array}\right)\left|C_{N}\right|^{2} \int d x_{M+1} \ldots d x_{N} \\
& \times\left|\psi_{N}(\underbrace{x, \ldots, x}_{M \text { terms }}, x_{M+1}, \ldots, x_{N})\right|^{2},
\end{aligned}
$$

which ceases to be the centroid distribution unless $N=M$. This result places stringent requirements on both the generation and the detection of nonclassical states for quantum lithography.

To properly define an intensity centroid measurement, on the other hand, first define the total photonnumber operator as $\hat{N} \equiv \int d x \hat{A}^{\dagger}(x) \hat{A}(x)$. Noting that $\left[\hat{N}, \int d x x \hat{A}^{\dagger}(x) \hat{A}(x)\right]=0$, the intensity centroid operator can be more generally defined as

$$
\hat{X} \equiv \frac{1}{\hat{N}} \int d x x \hat{A}^{\dagger}(x) \hat{A}(x),
$$

which can be measured by first recording the intensity pattern, discarding the results when the total photon number is zero, and calculating the centroid of the intensity pattern. The corresponding POVM is

$$
\begin{gathered}
\hat{\Upsilon}(X) \equiv \sum_{N=1}^{\infty} \int d \xi_{1} \ldots d \xi_{N-1} \hat{\Upsilon}\left(X, \xi_{1}, \ldots, \xi_{N-1}\right), \\
\hat{\Upsilon}_{0} \equiv|0\rangle\langle 0|, \quad \hat{\Upsilon}_{0}+\int d X \hat{\Upsilon}(X)=\hat{1} .
\end{gathered}
$$

The postselected intensity centroid distribution is then

$p_{m}(X) \propto \sum_{N=1}^{\infty}\left|C_{N}\right|^{2} \int d \xi_{1} \ldots d \xi_{N-1}\left|f_{N}\left(X, \xi_{1}, \ldots, \xi_{N-1}\right)\right|^{2}$,

which is the incoherent superposition of all Fock-state marginal centroid distributions. For mixed states, the distributions are simply the statistical average of pure-state results. If a quantum state has a small photon-number uncertainty and most Fock components have similar cen- 
troid distributions, the intensity centroid distribution, being an average of the Fock-state centroid distributions, would only be slightly smoothed by the incoherent superposition effect, whereas for quantum lithography, the effect of $N \neq$ $M$ can be more drastic $[4,6]$.

The centroid measurement can also be used to estimate the shift of an optical beam transverse position, due to deflection by a mirror in an atomic force microscope, for example. The results concerning the quantum uncertainty of the beam displacement obtained by Fabre et al. [9] can be understood using the theory proposed here. Making the strong mean-field approximation $\hat{A}(x)=A(x)+\Delta \hat{A}(x), \hat{N}$ and $\hat{X}$ can be linearized as $\hat{N}=N+\Delta \hat{N}$ and $\hat{X}=X+$ $\Delta \hat{X}$, where $\Delta \hat{N} \approx \int d x A^{*}(x) \Delta \hat{A}(x)+$ H.c., $\Delta \hat{X} \approx \frac{1}{N} \times$ $\int d x x A^{*}(x) \Delta \hat{A}(x)+$ H.c., H.c. denotes Hermitian conjugate, and $[\Delta \hat{N}, \Delta \hat{X}]=0 . \Delta \hat{X}$ becomes a quadrature operator that can be measured by homodyne detection with a local oscillator field $\propto x A^{*}(x)$. The theory presented in this Letter is in fact more general; it shows that repeated measurements of $\hat{X}$, without the linearization, can not only produce a Gaussian spot, but also complex quantum interference patterns like those in quantum lithography at the de Broglie resolution. For position tracking applications, such as atomic force microscopy, the proposed method can therefore accurately determine the position of a deflecting object even if the position significantly deviates from the mean. If the object is quantum, intensity centroid measurements also enable the imaging of its position wave function at the photonic de Broglie resolution.

Optical loss and imperfect detector efficiency are major issues for the use of nonclassical states for quantum imaging. For the specific case of a quantum Gaussian beam, the width of $p_{m}(X)$ after the beam propagates through a lossy channel can be analytically calculated using the quantum Langevin analysis described in Ref. [14]. Assuming $\langle\hat{X}\rangle=$ 0 without loss of generality, the result is

$$
\left\langle\Delta \hat{X}^{2}\right\rangle_{z}=\left\langle\Delta \hat{X}^{2}\right\rangle_{0}+\frac{\Delta x^{2}}{\eta N_{z}}\left(1-\eta e^{-\alpha z}\right),
$$

where $\left\langle\Delta \hat{X}^{2}\right\rangle$ is the centroid variance, the subscript $(z$ or 0 ) denotes the propagation distance, $N_{z}=N_{0} e^{-\alpha z}$ is the reduced photon number, $\Delta x^{2} \equiv\left\langle N^{-1} \int d x x \hat{A}^{\dagger}(x) \hat{A}(x)\right\rangle$ is the classical beam width squared, given by

$$
\Delta x^{2}=\frac{1}{4 \Delta k^{2}}\left[\frac{R_{0}}{N_{0}}+\frac{\left(1-1 / N_{0}\right)^{2}}{1-1 /\left(N_{0} R_{0}\right)}\right],
$$

$\eta$ is the detector efficiency, $\alpha$ is the power loss coefficient, and $\Delta k \sim 4 \pi \sin \theta / \lambda$ is the root-mean-square momentum bandwidth. $\Delta x$ and $\Delta k$ remain constant in a lossy channel [14]. $R_{0} \equiv 4 N_{0} \Delta k^{2}\left\langle\Delta \hat{X}^{2}\right\rangle_{0}$ is the initial normalized centroid variance, with $R_{0}=1$ at the standard quantum limit (SQL) for classical Gaussian beams and $R_{0}=1 / N_{0}$ at the "Heisenberg" limit. In the classical case, $R_{0}=1, \Delta x=$
$1 /(2 \Delta k),\left\langle\Delta X^{2}\right\rangle_{z}=\Delta x^{2} /\left(\eta N_{z}\right)$, and the centroid variance remains at the SQL for the detected photon number. Close to the "Heisenberg" limit, however, $\Delta x$ becomes much larger than $1 /(2 \Delta k)$, and the increase in centroid variance becomes much more sensitive to loss. As long as $R_{0} \gg$ $1 / N$, quantum imaging by intensity centroid measurements is fairly robust to loss. The effect of loss for more complex quantum images may be similarly studied using the Langevin method, but is beyond the scope of this Letter.

Given the recent progress in the spatial engineering of biphoton states $[4,5]$ and photon-counting detector technology, the proposed method is expected to be immediately realizable using current technology, at least for $N=2$. For $N>2$, it is more challenging to create the required nonclassical states of light. The proposed method nonetheless greatly simplifies the detection of multimode nonclassical optical states, and should be useful, at the very least, as a diagnostic tool in the study of quantum physics.

Discussions with Jeffrey Shapiro, Seth Lloyd, Lorenzo Maccone, and Vittorio Giovannetti are gratefully acknowledged. This work is financially supported by the Keck Foundation for Extreme Quantum Information Theory.

*mankei@mit.edu

[1] J. Jacobson et al., Phys. Rev. Lett. 74, 4835 (1995).

[2] M. Born and E. Wolf, Principles of Optics (Cambridge University Press, Cambridge, UK, 1999).

[3] A. N. Boto et al., Phys. Rev. Lett. 85, 2733 (2000).

[4] G. S. Agarwal et al., Phys. Rev. Lett. 86, 1389 (2001); S. J. Bentley and R. W. Boyd, Opt. Express 12, 5735 (2004); R. W. Boyd and S. J. Bentley, J. Mod. Opt. 53, 713 (2006), and references therein; P. R. Hemmer et al., Phys. Rev. Lett. 96, 163603 (2006).

[5] M. D'Angelo, M. V. Chekhova, and Y. Shih, Phys. Rev. Lett. 87, 013602 (2001).

[6] G. Björk, L. L. Sánchez-Soto, and J. Söderholm, Phys. Rev. Lett. 86, 4516 (2001); Phys. Rev. A 64, 013811 (2001).

[7] C. Thiel et al., Phys. Rev. Lett. 99, 133603 (2007).

[8] V. Giovannetti et al., Phys. Rev. A 79, 013827 (2009).

[9] C. Fabre, J. B. Fouet, and A. Maître, Opt. Lett. 25, 76 (2000); N. Treps et al., Phys. Rev. Lett. 88, 203601 (2002); N. Treps et al., Science 301, 940 (2003); S. M. Barnett, C. Fabre, and A. Maître, Eur. Phys. J. D 22, 513 (2003).

[10] M. Tsang, Phys. Rev. A 75, 043813 (2007).

[11] M. Tsang, Phys. Rev. Lett. 101, 033602 (2008).

[12] V. Giovannetti, S. Lloyd, and L. Maccone, Nature (London) 412, 417 (2001); V. Giovannetti et al., Phys. Rev. Lett. 88, 183602 (2002); O. Kuzucu et al., ibid. 94, 083601 (2005).

[13] J. B. McGuire, J. Math. Phys. (N.Y.) 5, 622 (1964); Y. Lai and H. A. Haus, Phys. Rev. A 40, 844 (1989); 40, 854 (1989); M. Tsang, Phys. Rev. Lett. 97, 023902 (2006).

[14] M. Tsang, Phys. Rev. A 75, 063809 (2007). 